\title{
Structural Health Monitoring of Wind Turbine Blades: Acoustic Source Localization Using Wireless Sensor Networks
}

\author{
Omar Mabrok Bouzid, ${ }^{1}$ Gui Yun Tian, ${ }^{2}$ Kanapathippillai Cumanan, ${ }^{3}$ and David Moore ${ }^{4}$ \\ ${ }^{1}$ Faculty of Engineering, Al-Jabel Al-Garbi University, Gharian, Libya \\ ${ }^{2}$ School of Electrical and Electronic Engineering, Newcastle University, Newcastle upon Tyne NE1 TRU, UK \\ ${ }^{3}$ Department of Electronics, University of York, York YO10 5DD, UK \\ ${ }^{4}$ School of Engineering and the Built Environment, Glasgow Caledonian University, Cowcaddens Road, Glasgow G4 OBA, UK
}

Correspondence should be addressed to Omar Mabrok Bouzid; drombouzid@gmail.com

Received 4 August 2014; Revised 14 December 2014; Accepted 15 December 2014

Academic Editor: Xiaowei Ye

Copyright (C) 2015 Omar Mabrok Bouzid et al. This is an open access article distributed under the Creative Commons Attribution License, which permits unrestricted use, distribution, and reproduction in any medium, provided the original work is properly cited.

\begin{abstract}
Structural health monitoring (SHM) is important for reducing the maintenance and operation cost of safety-critical components and systems in offshore wind turbines. This paper proposes an in situ wireless SHM system based on an acoustic emission (AE) technique. By using this technique a number of challenges are introduced due to high sampling rate requirements, limitations in the communication bandwidth, memory space, and power resources. To overcome these challenges, this paper focused on two elements: (1) the use of an in situ wireless SHM technique in conjunction with the utilization of low sampling rates; (2) localization of acoustic sources which could emulate impact damage or audible cracks caused by different objects, such as tools, bird strikes, or strong hail, all of which represent abrupt AE events and could affect the structural health of a monitored wind turbine blade. The localization process is performed using features extracted from aliased AE signals based on a developed constraint localization model. To validate the performance of these elements, the proposed system was tested by testing the localization of the emulated AE sources acquired in the field.
\end{abstract}

\section{Introduction}

Offshore wind turbines are often situated in remote areas which are difficult to reach. Faults can cause significant downtime and are often costly to address. In order to meet these challenges turbines are being combined with structural health monitoring (SHM) systems for continuous online health monitoring. This form of monitoring helps in detecting abnormal changes in structural health so that prompt maintenance can be carried out. It also enables engineering inspectors to easily monitor turbines from offsite locations without any safety risks $[1,2]$. Incorporating SHM techniques in many applications ranging from civil to engineering applications such as bridges $[3,4]$ and wind turbines provides significant advantages. These include minimizing operation and maintenance costs as well as the ability to collect information and data for future design and optimisation of blades. However, for wind turbine blades, using these techniques has some associated challenges due to the remote location of the turbines and the accessibility to them during extreme weather conditions. In situ monitoring approaches for wind turbines play a key role in addressing those challenges as they can help in detecting faults while the turbines are in operation, allowing appropriate action to be taken in time to avoid further damage or failure.

Recently, there have been some attempts to extend laboratory methods as in situ monitoring systems while the blades are static and in service [5-10]. These in situ techniques show potential for wind turbine blade inspection. Nevertheless, such techniques may be costly, time-consuming, relying on regular inspection intervals, and needing to be supervised by an operator which makes them impractical for offshore wind turbines. In contrast, wireless passive inspection techniques could be a more appropriate solution for providing an in situ monitoring. The main reasons for this are that a large number of wind farms are being placed in remote areas, and the size of the turbines has become physically larger. These factors make their transportation and reaching them for inspection very 
difficult. Thus, the selection of suitable passive techniques and the integration of several different technologies are important to develop a robust continuous online SHM system [11].

This has motivated the proposal of an in situ SHM system for wind turbine blades based on the integration of wireless sensor networks (WSNs) and acoustic emission (AE) technology. AE is a passive technique that can be applied to locate and assess complex composite structures [12]. For wind turbine blade, the $\mathrm{AE}$ allows the investigation of defects in their structure under wind load while the wind turbine is in service. In addition, most types of blade failure cause detectable AE waves, including crack initiation and growth and crack opening as well as closure [13].

WSNs also provide an ideal means for sending AE sensing data to a remote control unit. The integration of these two technologies in this application, however, faces several challenges. The large size of the wind turbine blades requires the deployment of a considerable number of AE sensors to achieve full blade coverage. It is also necessary that the proposed system should be able to sense the condition of the blades' structure all of the time. Such conditions lead to the generation of enormous amounts of sensing data which would overload the resources of WSNs in terms of data transmission and memory space.

Thus, the monitoring system proposed in this paper is employed in conjunction with the novel concept of utilizing low sampling rates in acquiring acoustic signals, discussed in [14], so that a significant reduction of data can be achieved during the monitoring process of wind turbine blades. In [15], the novelty of using such system in monitoring environmental conditions using different AE features extracted from the aliased versions on-board has been validated. This paper aims to use AE features such as arrival time and peak amplitude also extracted from the aliased versions on-board for locating AE sources. To the authors' knowledge, no in situ wireless SHM system for wind turbine blades has yet been developed with use of low sampling rates below the Nyquist criterion to locate AE sources. The proposed system is validated using the in situ wireless system deployed on wind turbine blades. The main contributions of this paper are as follows:

(i) Through the implementation of an in situ SHM system on a $300 \mathrm{~W}$ wind turbine installed at Newcastle University, the concept of utilizing low sampling rates in the acquisition operation of AE signals has been evaluated in a real world scenario.

(ii) The use of features extracted from aliased AE signals to localize emulated AE sources in conjunction with a localization model developed for the complex structure of wind turbine based on a constraint geometrical point localization technique. To validate the performance, the proposed system has been tested and precise source localization results have been achieved.

The paper is structured as follows. In Section 2, source localization methods using low sampled data are discussed for locating artificial AE sources on wind turbine blades. The in situ wireless SHM system for monitoring wind turbine blades is discussed in Section 3. Section 4 presents the strategies of acquiring $\mathrm{AE}$ events and $\mathrm{AE}$ feature extraction when performing field studies. In Section 5, the results of AE source localization at low sampling rates are presented. Finally, conclusion is laid out in Section 6.

\section{Source Localization on Wind Turbine Blades}

Acoustic source localization using WSNs has a number of possible applications, including intruder detection, sniper localization [16]. Wang et al. [17] and Saric et al. [18] present an overview of such wireless localization systems with detailed analyses and characterisations of typical source localization algorithms in WSNs. In [19], a PZT-based wireless system for composite impact monitoring is proposed to give a localized search area for further inspection. The wireless $\mathrm{AE}$ source localization technique, proposed in the course of this paper, is intended to be used for SHM of wind turbine blades. It differs from work in the aforementioned studies in the sense that it utilises aliased versions of $\mathrm{AE}$ signals instead of the original signals in the localization process. Therefore, the results presented in [17-19] would not be appropriate for a fair comparison.

In contrast to the work presented in [20], the calculations of localization features such as arrival time and peak amplitude are locally extracted by the individual acoustic wireless units. These features are then transferred to the control unit where they are assembled and fused to estimate the location of $\mathrm{AE}$ events.

A line-of-sight localization is conducted in order to measure the velocity with which $\mathrm{AE}$ signals propagate in the wind turbine blades. In addition, it is used to verify the possibility of locating AE events based on their arrival times extracted by the individual wireless units from the aliased versions. The wind turbine blade is made of carbon fibre and artificial AE events are generated by hitting the blade with a metallic ball at known positions between the sensors. The ball has a diameter of $9 \mathrm{~mm}$ and a mass of $3 \mathrm{~g}$.

It is important for the experiments conducted that the AE events are traced in order to specify the active zone or blade (i.e., the damaged blade). This is useful so that more attention can be given to that particular zone or blade. This can be done by a zonal localization, where each blade is assigned to an $\mathrm{AE}$ wireless unit and via a comparison between the maximum amplitudes of AE signals received at the wireless units, the active blade can be specified. To trace the active region within a large structure of wind turbine blade (i.e., in twodimensional position), AE wireless units can be spaced apart on the blade and AE sources can be assumed to be within a region and less than halfway between the wireless units. In this case, methods explained in [21] can be applied to trace the AE source. In this paper, the zonal localization is used to specify the active blade, and the results of this localization are presented in Section 5. Constraint geometrical point location technique is used to pinpoint precisely the AE source based on the arrival time and the predetermined sound velocity $(v)$.

Due to the small size of the $300 \mathrm{~W}$ wind turbine used in this study, a combination of zonal and constraint geometrical 


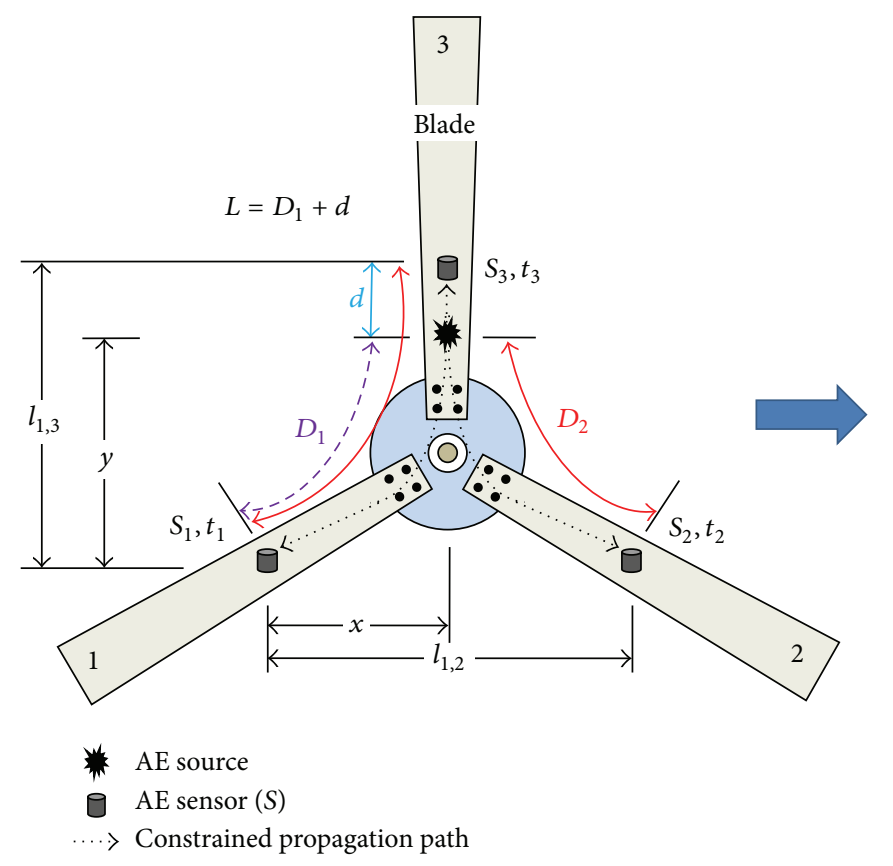

(a) Sensor configuration

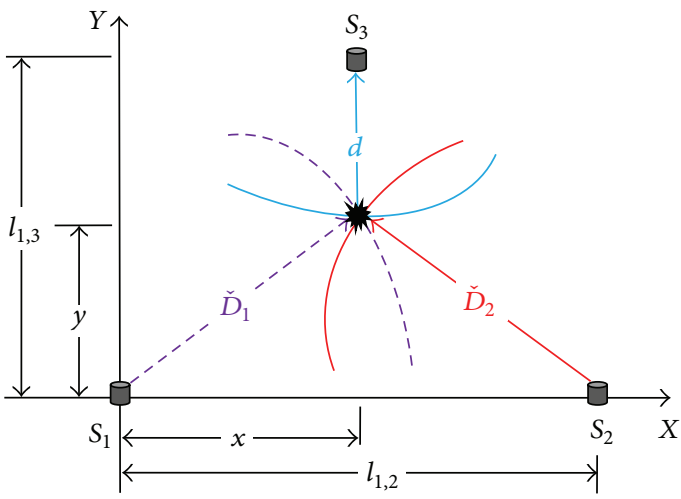

(b) $2 \mathrm{D}$ localization model

FIgURE 1: Proposed configuration of acoustic wireless units and 2D model: $x$ and $y$ are the unknown coordinates of AE source, $t_{i}$ is the arrival time of AE signal at AE sensor $S_{i}, d$ is the distance between $S_{3}$ and the AE source, and $l_{1,3}$ and $l_{1,2}$ are the approximated distances between $S_{1}$ and $S_{3}$ as well as $S_{1}$ and $S_{2}$, respectively.

point localization is implemented as shown in Figure 1(a). To apply this localization technique to the wind turbine blades, a model of $2 \mathrm{D}$ localization needs first to be developed. To achieve this, $\mathrm{AE}$ sensors are assumed to be configured as shown in Figure 1(a). In this configuration, it is also assumed that the $\mathrm{AE}$ sensors are located in a $2 \mathrm{D}$ plane and the $\mathrm{AE}$ signals generated propagate in constrained paths along the blades as denoted by dashed lines. In Figure 1(a), $D_{1}$ and $D_{2}$ represent the shortest paths which will be taken by the $\mathrm{AE}$ signals in order to reach the $\mathrm{AE}$ sensors $S_{1}$ and $S_{2}$, respectively.

Based on the configuration shown in Figure 1(a) and abovementioned assumptions, a $2 \mathrm{D}$ model is derived as shown in Figure 1(b) where the shortest paths $D_{1}$ and $D_{2}$ are approximated by $\check{D}_{1}$ and $\check{D}_{2}$, respectively. In this case, the basic principle of constraint geometrical point localization, explained in [22], is used to estimate the unknown coordinates of the AE source, $x$ and $y$, which are indicated by the intersection of hyperbolas which are not shown in this figure. The arrival time features of aliased $\mathrm{AE}$ signals are calculated using the first threshold crossing. This method is applied in conjunction with the extracted envelope to overcome the ambiguity caused by utilizing low sampling rates. It also leads to enhancing the detection accuracy of the arrival times of the captured aliased $\mathrm{AE}$ versions.

A simple time synchronization procedure is applied to overcome the drift in the clocks of the wireless units in which the wireless units time-stamp the arrival of a start sample command based on local times of the individual wireless units, which is denoted by $t_{b i}$. These times are affected by the drift in the individual clocks. Thus, to overcome such a drift these timestamps are converted to a global time according to the relation: global arrival time $=\left(t_{i}-t_{b i}\right)$, where $t_{i}$ is the local arrival time of the detected $\mathrm{AE}$ signal at each wireless unit and $i \in(1,2,3)$. A sequence of line-ofsight measurements has been conducted, as discussed above, in order to test the robustness of this method, in which the arrival times were calculated with and without using the global arrival time relation.

By comparing the results obtained from both cases, it has been found that using the above mentioned relation of global arrival time reduces the time synchronization errors from $46.33 \mu$ s to $1.55 \mu$ s which represents an average improvement of $96.65 \%$ according to the setting of the experiments conducted. The reason behind this enhancement is that the use of the global time instead of the local time minimises the effect of the drift in the clocks of the individual wireless units.

Such an improvement will have a positive impact on both velocity and localization measurements. Based on detected arrival times $t_{1}$ and $t_{2}$ of the detected AE signals, a calibration method is used to measure the velocity of wave propagation (v) using the relation: $v=L /\left(t_{2}-t_{1}\right)$, where $L$ is the known distance between the two AE sensors (i.e., wireless units). Due to the short length of the blade used, only a small number of measurements are conducted. However, to show the robustness and repeatability of these measurements, each one is repeated a number of times and the results are discussed in Section 5.

The proposed approach is examined by conducting several experiments to locate $\mathrm{AE}$ sources produced at a known 


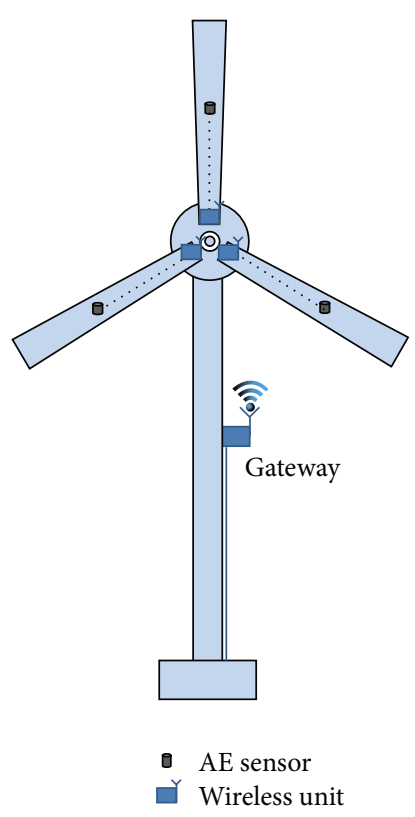

(a) System deployment

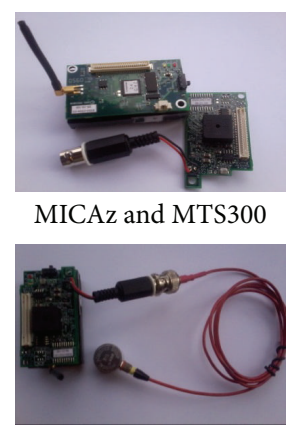

Wireless sensing node

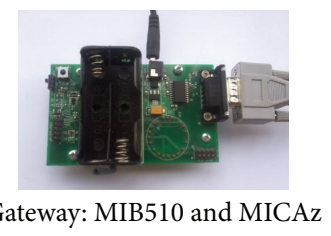

(b) Hardware components used

FIGURE 2: Wireless monitoring network installed on the top of a $300 \mathrm{~W}$ wind turbine.

location on blades by artificial AE events produced via hitting the blades with a plastic stick which has a diameter and length of $15 \mathrm{~mm}$ and $500 \mathrm{~mm}$, respectively. The intention of using such sound sources, instead of using pencil break test with graphite lead, is to emulate impact damage or audible cracks caused by different objects such as tools, bird strikes, or strong hail. The results of these experiments are discussed in Section 5. Next, the wireless SHM setup is discussed.

\section{In Situ Wireless SHM System for Wind Turbine Blades}

3.1. Components of Wireless Sensing Node. The development of the in situ SHM system takes advantage of wireless technology to form a monitoring network. This system is comprised of MICAz motes equipped with the sensor board (MTS310) which are used in developing the wireless system, as shown in Figure 2. MICAz motes represent one of the lowcost current state-of-the-art sensor technologies that provide capabilities to build wireless networks [23]. The MICAz is $2.4 \mathrm{GHz}$, IEEE/ZigBee 802.15 .4 boards equipped with $4 \mathrm{~KB}$ of RAM. At the centre of the mote is the processing core which contains function modules for data collection, processing, and communication control. The MPR2400 is based on the Atmel ATmega128L and capable of running at speeds up to $7.3 \mathrm{MHz}$. This mote also supports a 10-bit analogue to digital converter with 8 channels and $0-3 \mathrm{~V}$ input range.

The omnidirectional microphone sensor of the MTS310 board is replaced by a $\mathrm{BNC}$ connector for $\mathrm{AE}$ sensors, as shown in Figure 2(b). The AE sensor is the BII-7070 from "Benthowave Instrument Inc." (http://www.benthowave.com/) and operates at a usable frequency range of $0.1 \mathrm{~Hz}$ to $400 \mathrm{kHz}$ with a size of $\Phi \times L=18.6 \times 20 \mathrm{~mm}$. It is characterised by a relatively small mass and low cost.

All of the sensing units communicate with a PC base station via a radio-frequency interface and are programmed in a TinyOS environment [24]. The PC base station includes an MIB510 gateway board incorporating MICAz mote which is used to forward the extracted features or the raw data to the PC for monitoring and processing.

3.2. In Situ Wireless SHM System Deployment. The wireless prototype system is applied to a $300 \mathrm{~W}$ wind turbine which is installed on the roof of the School of Electrical and Electronic Engineering (EEE). This wind turbine has three blades made of carbon with a diameter of $1.5 \mathrm{~m}$. Each AE sensor takes charge of one blade of the wind turbine structure and records the AE events independently. Each unit is programed with a unique identification to prevent data chaos in data transmission.

Bringing together the entire component subsystems into one system, which is integrated into the wind turbine unit for in situ monitoring requires, ensures that the attachment of sensing units to the wind turbine blades will last for a long time. This is achieved by placing the wireless units in waterproofed plastic secured by cable tidy-ties in the wind turbine nose. This has the advantage that it makes the radius of rotation of the wireless units very small, so that the rotation of the wind turbine blades has no effect on the data transmitted. This is because the path loss is inversely proportional to the rotating radius of wireless units [25].

A diagram showing the attachment configuration is given in Figure 3, where a portion of a protection tube of $18.7 \mathrm{~mm}$ diameter and $24 \mathrm{~mm}$ in length is fixed by an Araldite adhesive 


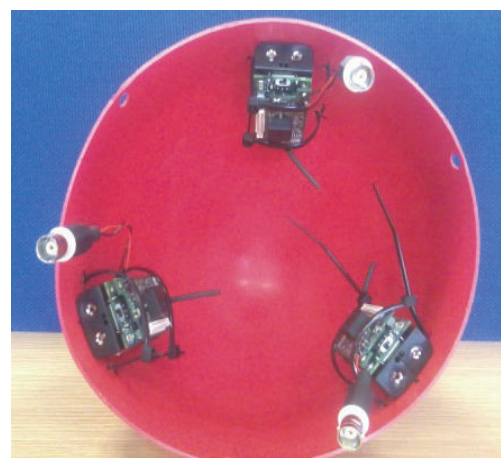

(a)

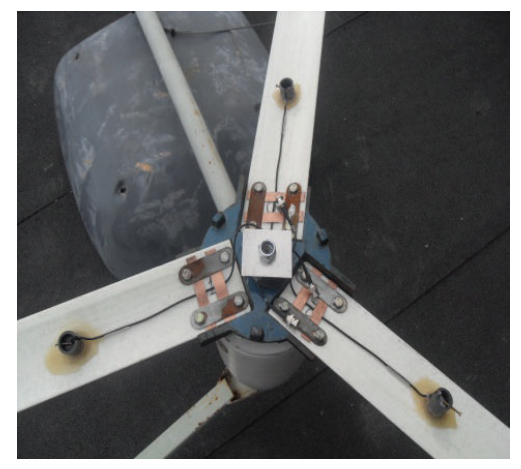

(b)

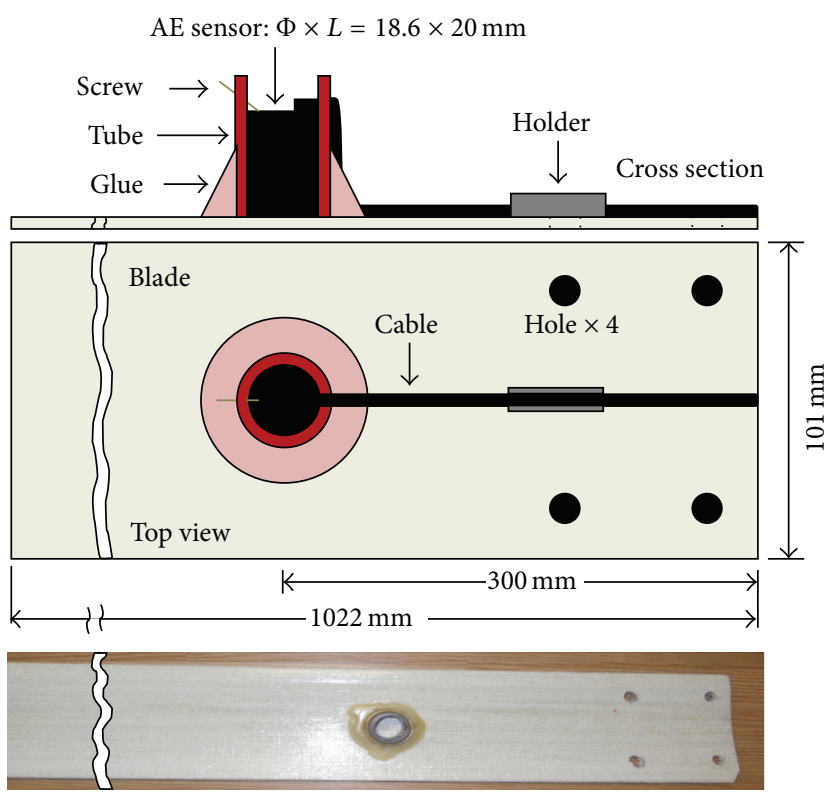

(c)

FIGURE 3: Wireless unit installation: (a) photograph of WSN node inside the nose, (b) photograph of acoustic emission sensors fixed on the blades, and (c) diagram of acoustic emission sensors fixed on the blades.

to the blade. The AE sensor is positioned in the tube and secured by a screw, and its cable is held by a cable holder which is fastened to the blade using the Araldite adhesive. The reason for this attachment is that it gives the system the flexibility to allow changes to be made to the AE sensor at any time and also minimises the noise generated compared to the use of tabs [26]. The AE sensor is attached at an equal distance of $300 \mathrm{~mm}$, which is almost $30 \%$ along the length of the blade from the root section. It has been found by both simulation and experimental methods for the fact that this area is more prone to damage [5].

The AE data captured from the rotating blades is sent through wireless medium to the MIB510 serial gateway which is attached to the wind turbine tower. This data can then be transferred to the remote control unit via wired or wireless communication, such as using Wi-Fi or any other wireless techniques. The study of limitation of wireless signal transmission which can be presented in real situations is out of the scope of this paper, having been considered in future work. In this application, the gateway forwards the collected data to the PC base station in the lab through a serial cable which is also used to provide power to the serial gateway.

The entire wind turbine unit was installed with the wireless SHM system on the School's roof. The gateway is placed in a waterproof case and attached next to the wind turbine tower, as discussed above. This gateway is basically used for sending a start sensing command and setting the threshold value of the sensing process as well as switching between the transmission modes discussed in the next section. These commands are initiated by a control unit which is located in the School's lab as discussed below.

To make communication between the control unit and the wireless sensing units easier and to control the wireless monitoring system, a software package for this system was designed. This package is run by a PC and it consists of an operating package, a Java application, and MATLAB scripts to provide a user-friendly graphical user interface (GUI). The former package is executed by the TinyOS operating system installed on the MICAz hardware platform, and its main function is to perform the operations of sensing and 


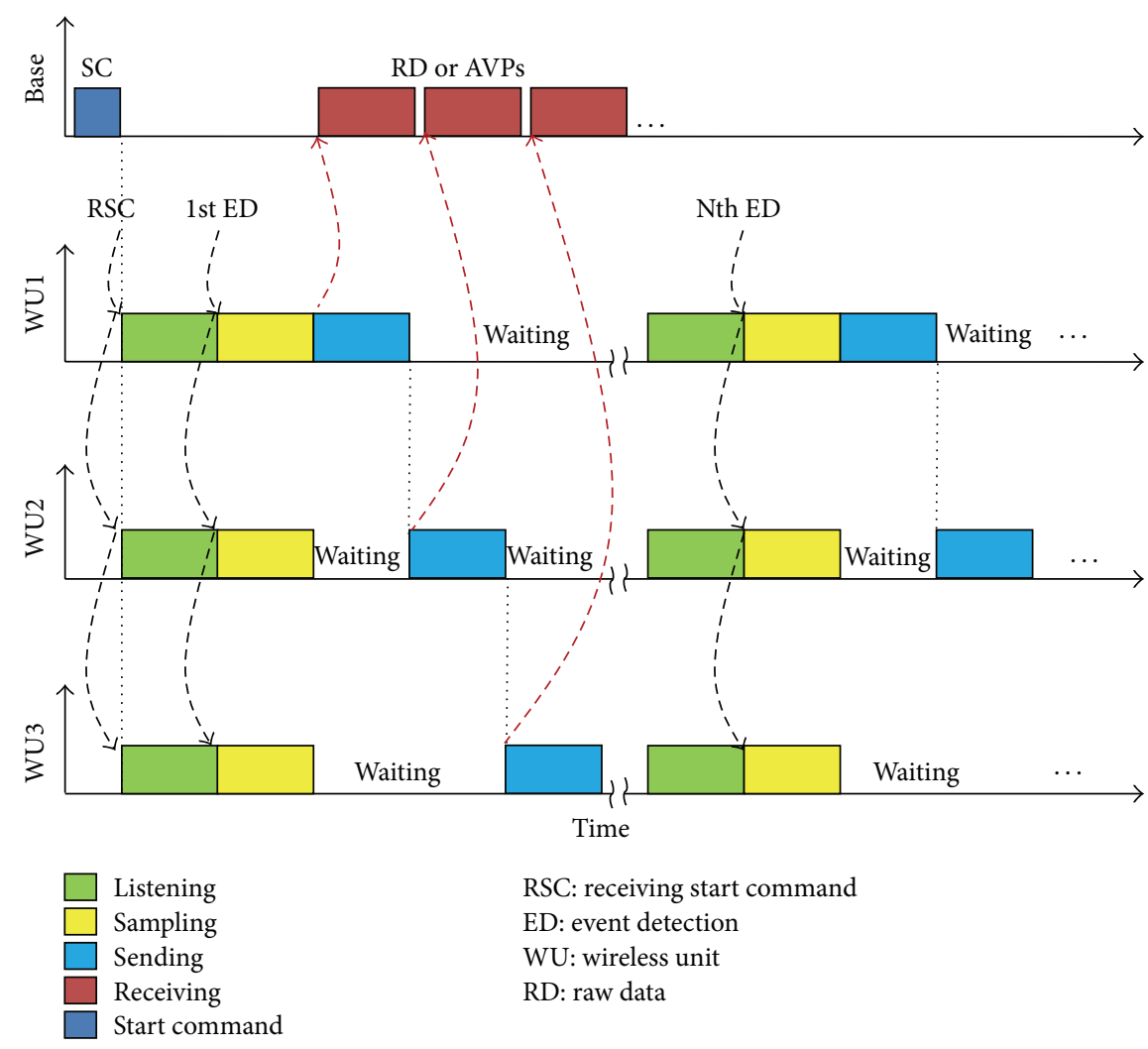

FIGURE 4: Schematic diagram of synchronised sensing and transmission operations.

the transmission of AE data. The GUI is used to control and interact with the developed wireless monitoring system.

\section{Field Study}

For field studies where continuous online monitoring obviously is required, the use of wireless sensing systems requires approaches for decentralised data processing (i.e., on-board the wireless units). In this case, important $\mathrm{AE}$ features can be locally extracted from the raw data and only those features to be sent to the collection point. Next, the strategies of acquiring $\mathrm{AE}$ events and feature extraction algorithm for the field study of this work are presented.

4.1. Synchronized Sensing and Transmission Operations. The sensing of the AE events, in this application, differs slightly from that explained in [20]. This process commences in all wireless sensing units with the receipt of a start command, indicated by "SC" in Figure 4. This command is broadcast by the base station and forces the wireless units to enter into a listening mode which makes them wait for AE events to occur which are above a preset threshold value, indicated by "ED." Once the wireless units detect such events they start acquiring AE signals at a sampling rate of almost $4.8 \mathrm{KHz}$ for a sampling period of $0.11 \mathrm{~s}$.

After finishing the sensing operation, the sensing units start to execute the sending operation based on the transmission mode as discussed below. As soon as they complete the sending operations, they enter again into the listening mode and a similar procedure is repeated in the same sequence.

In the proposed wireless system, the transmission operation is an event-based process, in which wireless units will not send any data unless an AE event is detected. Once the $\mathrm{AE}$ event is detected, these units will transfer their data after completing the sampling process according to the strategy explained in [20]. Note that the waiting period for each wireless unit is very small, due to the short time required for the transmission process. This is in particular for the transmission of acoustic vector properties (AVPs) which incorporate features extracted from the aliased $\mathrm{AE}$ versions, since this is the default transmission option as explained below. Thus, data loss will be rare and there will be no need to compensate for the data that is not captured during the waiting period of each sensor unit.

Moreover, depending on the transmission mode, the wireless units will send either raw data or the AVPs to the control unit. The default setting of this mode is set to AVP mode. In this case, the wireless units process the raw data in order to extract AE features from the aliased versions; and only those in the form of AVPs, instead of the aliased versions, will be sent to the remote control unit. For cases where the transmission mode is set to raw data, the wireless units will follow the same sending strategy explained in [20]. Irrespective of transmission mode, data are collected from 


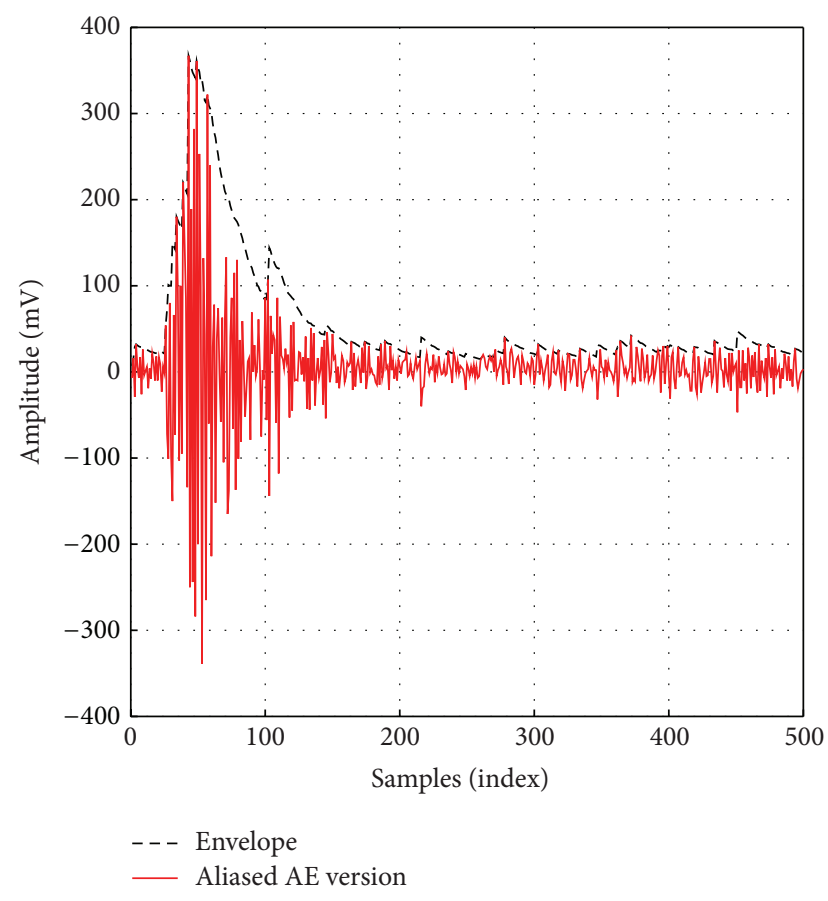

FIgURE 5: Aliased AE signal with its envelope.

various wireless units and aggregated into the control unit for further analysis.

\subsection{Signal Processing and AE Feature Extraction Algorithms.} The wireless system developed here is based on the use of MICAz platforms, which have limited power resources and low processing capabilities. Therefore, relatively simple signal processing algorithms need to be considered for onboard AE feature extraction. In addition, for better analysis and localization results, content-based features should be extracted from the envelope of the received $\mathrm{AE}$ aliased versions rather than from the amplitude values of the time domain signal. This is because envelopes optimise the signal shape and minimise the ambiguity in the signal caused by lowering the sampling rate [14]. The envelope of the aliased AE signal is extracted on-board as explained in [15], where relatively simple and not computationally complex algorithm is used.

Figure 5 shows an example of an aliased AE version with the envelope extracted using a one-pole filter. The aliased version was captured using the developed in situ SHM system.

As it is known $\mathrm{AE}$ waves are abnormal and transient activities, which are affected by the characteristics of the induced stress field. These waves can be converted into a number of useful AE parameters or features, as shown in Figure 6, which can then be used to identify these events. Some of these parameters are as follows [27]:

(i) Amplitude, $A$, is the maximum amplitude which is usually measured in decibels $(\mathrm{dB})$.

(ii) Rise time, $R$, is the time interval between the first threshold crossing and the time when the AE signal reaches its amplitude.

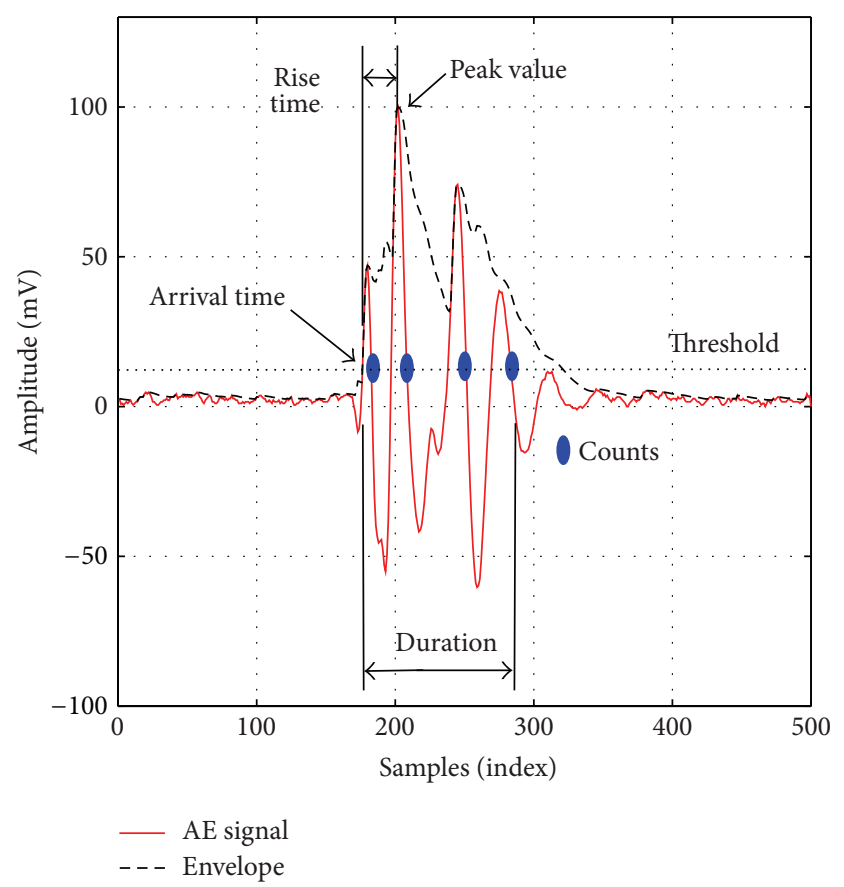

Figure 6: Key features of an aliased AE signal.

(iii) Duration, $D$, represents the time difference between the first and the last crossings of the threshold value.

(iv) Counts, $N$, refer to the number of times the signal crosses the threshold value.

(v) Power, $P$, is the area under the curve or the summation of the squared sample values.

(vi) Arrival time, $T$, is the time of the first threshold crossing.

(vii) Root mean square, RMS, is a statistical average parameter used for comparatively long transients.

One of the advantages of the utilization of low sampling rates is that the original shape can be conserved, which helps in maintaining the most salient information or features of the original $\mathrm{AE}$ signals without requiring to reconstruct them. This means that the most relevant $\mathrm{AE}$ event parameters, discussed above, will be retained if the aliased versions of acoustic signals are used. Fortunately, most of them are timedomain features. However, not all of the common AE features can be extracted from these versions. For example, counts are one of the exceptional $\mathrm{AE}$ features due to the reason that this feature represents the number of threshold crossings along the timespan between the initial and the last crossings of the threshold value. These in-between crossings are in most cases unavailable when envelopes are utilised in the extraction process. On the other hand, the duration feature which represents the timespan between these two points is still valid in this domain, since the threshold crossings occur at these points.

Other AE features, including peak value, arrival time, mean value, and power value, are extracted from the envelopes of the aliased AE signals. These features have the 
TABLE 1: Line-of-sight results of AE events localization based on AVPs.

\begin{tabular}{lccccc}
\hline Exp. $\#$ & $L(\mathrm{~cm})$ & $d(\mathrm{~cm})$ & $\begin{array}{c}\text { Average of estimated } d \\
(\mathrm{~cm}) \text { from 6 readings }\end{array}$ & STD of $d$ & Error $(\%)$ \\
\hline 1 & & 35 & 35.52 & 0.69 & 1.49 \\
2 & 82 & 20 & 19.65 & 1.23 & 0.36 \\
3 & & 15 & 13.47 & 0.57 & 1.75 \\
\hline 4 & & 40 & 40.03 & 1.03 & 0.20 \\
5 & 88 & 30 & 31.52 & 1.55 & 5.07 \\
6 & & 21 & 22.30 & 6.19 \\
\hline
\end{tabular}

advantage that they can be computed while the sensing process is in progress without the need to have acquired the whole signal first. The extracted features transferred to the control unit where they are characterised and used to localize the $\mathrm{AE}$ events, as discussed in the next section.

\section{Results and Discussion}

To evaluate the new concept for the sensing technique proposed in [14] for field test studies, wireless localization measurements were conducted using the in situ wireless SHM system discussed in Section 3. In these measurements, locations of $\mathrm{AE}$ sources were estimated based on the use of features locally extracted from aliased AE signals. The following subsections discuss the functionality of this system in terms of the localization of $\mathrm{AE}$ events on the wind turbine blades caused by artificial AE sources via hitting the blades with the same plastic stick discussed in Section 2. Such an AE source could emulate impact damage or audible cracks caused by different objects, such as tools, bird strikes, or strong hail, all of which represent abrupt AE events.

5.1. Line-of-Sight Localization of Events. Different artificial $\mathrm{AE}$ events were generated at known positions and localized using line-of-sight localization on a single wind turbine blade. The results show the potential of the AE source localization at low sampling rates based on the arrival time feature. The relation $d=0.5 \cdot\left(L-v \cdot \Delta t_{21}\right)$ is used to localize the $\mathrm{AE}$ source, where $d$ is the distance from first hit AE sensor, $v$ is the velocity measured, and $\Delta t_{21}$ is the difference in arrival times between AE sensors. The results gained from six experiments are summarised in Table 1.

From the results shown in Table 1, the actual and estimated results are closely matched giving an overall error of $4.13 \%$ based on the measurement setting. This percentage error was calculated according to

$$
\text { Error } \%=\frac{\mid \text { measured value }- \text { actual value } \mid}{\text { actual value }} \cdot 100 \%
$$

As seen in Table 1, the rate of estimated error decreases as the distance $d$ increases. This is because the use of low sampling rates led to decreasing spatial resolution, which in contrast increases the error in the detection of arrival times of the signals as $d$ becomes smaller. In addition and based on these measurements, there is no effect of sampling speed of data acquisition on the precision of the AE localization.

5.2. Active Blade Identification. Active (damaged) blade identification has a significant importance in the application in SHM of offshore wind turbine blades. This is because it can restrict the inspection process to that particular blade, which saves cost and time. Figure 7 shows the AE aliased signals received as a consequence of hitting the three blades individually while they were static. Each reading consists of 500 samples and all of them are initiated by a simple threshold crossing condition $(100 \mathrm{mv})$, which was chosen experimentally to be above noise level. Figure 7(a) illustrates the results of hitting blade number one. It is clear that a strong AE event was occurring at this blade, which needs to be taken into account in event detection and localization as discussed next. The other two plots show the results of the propagation of this event to blades two and three.

In contrast Figures 7(b) and 7(c) depict the consequence of hitting blades two and three, respectively. In both cases, the strongest aliased AE signal can be used to specify which blade was subject to the hit, whereas weak signals were obtained in each case as a result of the propagation of both strongest aliased AE signals to the other blades. In addition, it can be observed in Figure 7(a) that there is a slight difference between the waveforms shown in this figure and those in Figures 7(b) and 7(c) in which the first shows that there is a minor hit result following the main hit result.

These aliased signals shown in Figure 7 also demonstrate that applying uniform sampling rates lower than Nyquist requirements in the acquisition process can still preserve the shape or contents of the AE signals. The shape and common $\mathrm{AE}$ features of these aliased versions are correlated with the AE signals captured at high sampling rates [27]. This is a very important criterion in the extraction of AE features in SHM applications, which is not satisfied in techniques previously applied where a set of random projected samples was used [28].

The determination of the active blade can also be achieved based on the AVPs received, as depicted in Figure 8 which summarises the results illustrated in Figure 7. For each test, the maximum peak values indicate the active blade which was hit. This means that, from Figure 8, and based on the peak values, the first test shows that the AE event occurred on blade number one, whereas they occurred on blades two and 

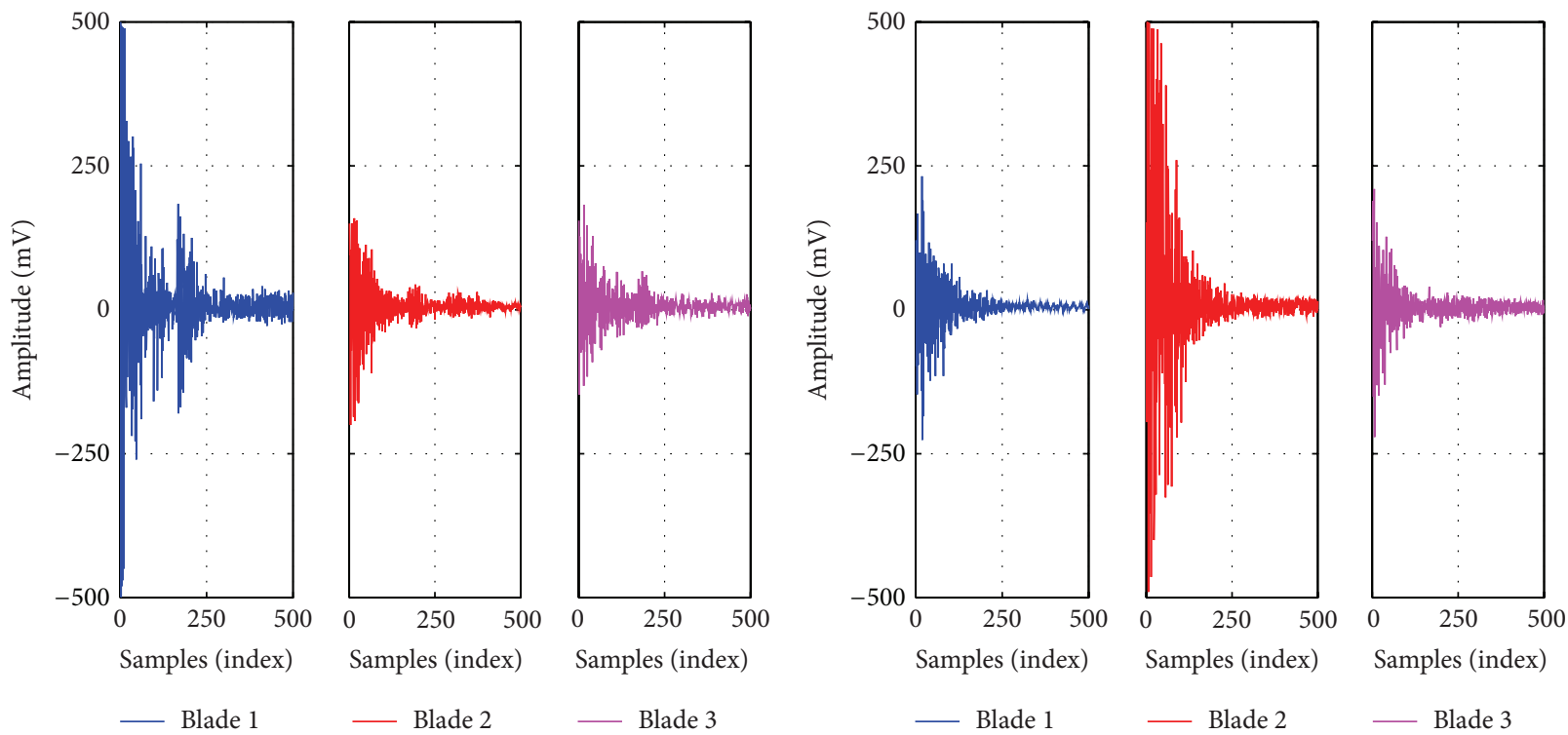

(a)

(b)
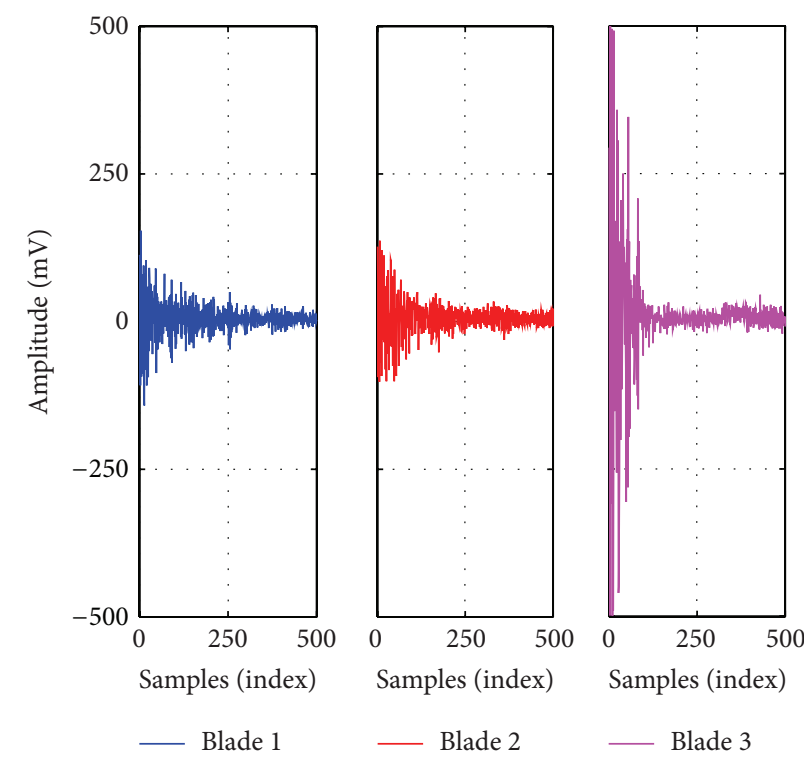

(c)

FIGURE 7: Aliased AE signals received from the wireless units as a consequence of hitting the three blades individually: (a) one, (b) two, and (c) three.

three in the second and third tests, respectively. The results gained here can be combined with those of the constraint geometrical point location technique to provide more precise source localization results for large scale wind turbine blades, as discussed next.

\subsection{Constraint Geometrical Point Localization of AE Events.} Due to the symmetry of the sensor configuration in Figure 1(a), the distance $x$ is known and equals $l_{1,2} / 2$ and the arrival times at $S_{1}$ and $S_{2}$ are equal, which means that $\Delta t_{21}=$ 0 . Thus, any two sensors located on the propagation path are sufficient to estimate the distance $d$ and consequently the coordinate $y$. In addition, once the active blade is specified based on zonal location, as explained in Section 2, then the proposed model in Figure 1(b) can be adjusted accordingly.

The proposed approach is examined by conducting wireless experiments to locate $\mathrm{AE}$ sources produced at known locations on blades by artificial AE events. For this purpose, the locations of $\mathrm{AE}$ sensors were changed to $50 \mathrm{~cm}$ instead of $30 \mathrm{~cm}$ on each blade, in order to provide more scope for changing the value of $d$. In these measurements, a calibration method as discussed in Section 2 is used to measure the velocity of wave propagation from the measured time difference between the different $\mathrm{AE}$ sensors on the blades, and it is found to be around $2 \times 10^{4} \mathrm{~m} / \mathrm{s}$ on average. It is worth mentioning that as the distance between the two AE sensors decreases the 
TABLE 2: Estimation results of constraint geometrical point localization.

\begin{tabular}{|c|c|c|c|c|c|c|}
\hline Exp. \# & $l_{1,2}, l_{1,3}(\mathrm{~cm})$ & $L(\mathrm{~cm})$ & $d(\mathrm{~cm})$ & $\begin{array}{l}\text { Average of estimated } d \\
(\mathrm{~cm}) \text { from } 6 \text { readings }\end{array}$ & STD of $d$ & Error (\%) \\
\hline 1 & \multirow{6}{*}{92} & \multirow{6}{*}{103} & 15 & 17.58 & 0.55 & 17.20 \\
\hline 2 & & & 20 & 21.70 & 1.68 & 8.50 \\
\hline 3 & & & 25 & 23.19 & 0.39 & 7.24 \\
\hline 4 & & & 30 & 31.90 & 1.50 & 6.33 \\
\hline 5 & & & 35 & 36.60 & 0.91 & 4.57 \\
\hline 6 & & & 40 & 41.61 & 0.74 & 4.02 \\
\hline
\end{tabular}

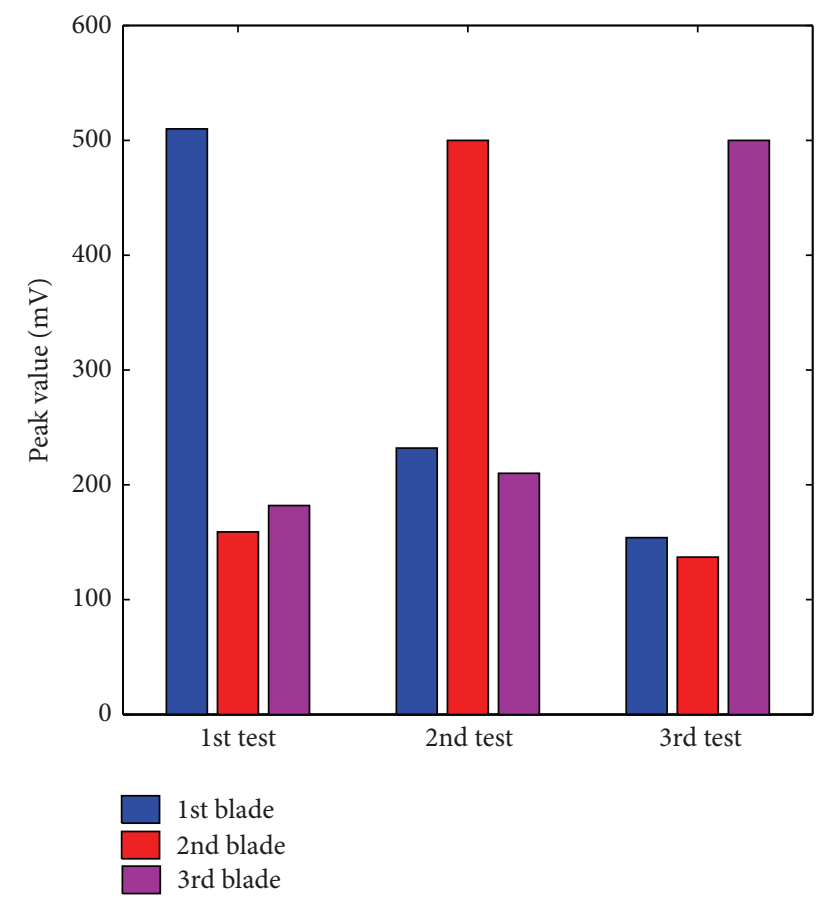

FIGURE 8: Active blade identification based on peak value features of AE events shown in Figure 7.

measured velocity becomes inaccurate. The reason behind this is that the velocity measurement will be dominated by the small distance between the two sensors which is dominated by the complex structure of the wind turbine at hub, whereas as this distance increases, it will be dominated by the simple one-dimensional structures of the blades.

The localization results obtained are summarised in Table 2. From these results, the actual and the estimated results are closely matched. They give an overall error of $7.98 \%$, which shows the feasibility of the proposed model for AE source localization at sampling rates much lower than the Nyquist rate for the specific wind turbine structure. The percentage error was calculated according to (1). It is noticeable that the percentage error also increases with a decrease in $d$. This is because as the $d$ decreases the difference in arrival times of signals at AE sensors decreases as well; therefore, estimation errors increase. In addition, the results obtained here show a systematic error within $2 \mathrm{~cm}$ which is most likely due to the gap between the $\mathrm{AE}$ sensors and wind turbine blades. These results show also that the sampling speed of data acquisition does not harm the precision of the AE localization. Such results, though for a small wind turbine system, show significant potential to be extended to largescale offshore wind turbine blades.

\section{Conclusions}

In this paper, the implementation of an in situ SHM system for wind turbine blades was introduced and described based on the integration of the AE technique and wireless technology. The developed system was integrated onto a $300 \mathrm{~W}$ wind turbine installed on the roof of Newcastle University. This system was used to evaluate a promising low sampling sound localization technique proposed in previous work. This was achieved by utilizing the content-based features extracted from the aliased AE signals on-board the wireless units to locate AE sources which emulated impact damage.

In addition, a localization model for constraint geometrical point localization technique was developed and applied to the three wind turbine blades. Wireless localization measurements were carried out to assess the localization capabilities of the proposed model on the wind turbine blades. The results show the feasibility of this model for AE source localization at sampling rates much lower than the Nyquist rate in this specific wind turbine structure.

Such conclusions are important for the further design and development of wireless SHM systems and their application in developing continuous in situ offshore large scale wind turbine blades condition monitoring systems. Further studies of this work will consider AE features with low sampling rate that can be used for the damage identification.

\section{Conflict of Interests}

The authors declare that there is no conflict of interests regarding the publication of this paper.

\section{Acknowledgments}

The authors would like to thank the FP7 HEMOW Project, "Health Monitoring of Offshore Wind Farms" (FP7-PEOPLE2010-IRSES, 269202, http://www.hemow.eu/), for funding the work. The authors would also like to express their gratitude to all the people from both mechanical and electronic workshops (School of Electrical and Electronic Engineering, 
Newcastle University) who have given their full support in making this implementation a success.

\section{References}

[1] P. J. Schubel, R. J. Crossley, E. K. G. Boateng, and J. R. Hutchinson, "Review of structural health and cure monitoring techniques for large wind turbine blades," Renewable Energy, vol. 51, pp. 113-123, 2013.

[2] Z. Hameed, Y. S. Hong, Y. M. Cho, S. H. Ahn, and C. K. Song, "Condition monitoring and fault detection of wind turbines and related algorithms: a review," Renewable and Sustainable Energy Reviews, vol. 13, no. 1, pp. 1-39, 2009.

[3] Y. Q. Ni, X. W. Ye, and J. M. Ko, "Monitoring-based fatigue reliability assessment of steel bridges: analytical model and application," Journal of Structural Engineering, vol. 136, no. 12, pp. 1563-1573, 2010.

[4] X. W. Ye, Y. Q. Ni, K. Y. Wong, and J. M. Ko, "Statistical analysis of stress spectra for fatigue life assessment of steel bridges with structural health monitoring data," Engineering Structures, vol. 45, pp. 166-176, 2012.

[5] C. C. Ciang, J.-R. Lee, and H.-J. Bang, "Structural health monitoring for a wind turbine system: a review of damage detection methods," Measurement Science and Technology, vol. 19, no. 12, Article ID 122001, 20 pages, 2008.

[6] J.-R. Lee, H.-J. Shin, C. C. Chia, D. Dhital, D.-J. Yoon, and Y.-H. Huh, "Long distance laser ultrasonic propagation imaging system for damage visualization," Optics and Lasers in Engineering, vol. 49, no. 12, pp. 1361-1371, 2011.

[7] C. S. Shin, B. L. Chen, and S. K. Liaw, "An FBG-based impact event detection system for structural health monitoring," Advances in Civil Engineering, vol. 2010, Article ID 253274 , 8 pages, 2010.

[8] N. Elkmann, T. Felsch, and T. Förster, "Robot for rotor blade inspection," in Proceedings of the 1st International Conference on Applied Robotics for the Power Industry (CARPI '10), pp. 15, Montreal, Canada, October 2010.

[9] P. Meinlschmidt and J. Aderhold, "Thermographic inspection of rotor blades," in Proceedings of the 9th European Conference on NDT, Berlin, Germany, 2006.

[10] N. Tralshawala and W. I. Faidi, "System and method for inspecting a wind turbine blade," United States Patent 8120522, 2011.

[11] I. Amenabar, A. Mendikute, A. López-Arraiza, M. Lizaranzu, and J. Aurrekoetxea, "Comparison and analysis of non-destructive testing techniques suitable for delamination inspection in wind turbine blades," Composites Part B: Engineering, vol. 42, no. 5, pp. 1298-1305, 2011.

[12] F. Ciampa and M. Meo, "A new algorithm for acoustic emission localization and flexural group velocity determination in anisotropic structures," Composites Part A: Applied Science and Manufacturing, vol. 41, no. 12, pp. 1777-1786, 2010.

[13] M. Huang, L. Jiang, P. K. Liaw, C. R. Brooks, R. Seeley, and D. L. Klarstrom, "Using acoustic emission in fatigue and fracture materials research," JOM, vol. 50, pp. 1-14, 1998.

[14] O. M. Bouzid, G. Y. Tian, J. Neasham, and B. Sharif, "Investigation of sampling frequency requirements for acoustic source localisation using wireless sensor networks," Applied Acoustics, vol. 74, no. 2, pp. 269-274, 2013.

[15] O. M. Bouzid, G. Y. Tian, K. Cumanan, and J. Neasham, "Wireless $\mathrm{AE}$ event and environmental monitoring for wind turbine blades at low sampling rates," in Proceedings of the World Conference on Acoustic Emission-2013: Advances in Acoustic Emission Technology, G. Shen, Z. Wu, and J. Zhang, Eds., vol. 158 of Springer Proceedings in Physics, p. 400, 2015.

[16] Á. Lédeczi, A. Nádas, P. Völgyesi et al., "Countersniper system for urban warfare," ACM Transactions on Sensor Networks, vol. 1, pp. 153-177, 2005.

[17] M. Wang, L. Ci, P. Zhan, and Y. Xu, "Acoustic source localization in wireless sensor networks," in Proceedings of the Workshop on Intelligent Information Technology Application (IITA '07), pp. 196-199, December 2007.

[18] Z. M. Saric, D. D. Kukolj, and N. D. Teslic, "Acoustic source localization in wireless sensor network," Circuits, Systems, and Signal Processing, vol. 29, no. 5, pp. 837-856, 2010.

[19] P. Liu, S. Yuan, and L. Qiu, "Development of a PZT-based wireless digital monitor for composite impact monitoring," Smart Materials and Structures, vol. 21, no. 3, Article ID 035018, 2012.

[20] O. M. Bouzid, G. Y. Tian, J. Neasham, and B. Sharif, "Envelope and wavelet transform for sound localisation at low sampling rates in wireless sensor networks," Journal of Sensors, vol. 2012, Article ID 680383, 9 pages, 2012.

[21] R. Gangadharan, G. Prasanna, M. R. Bhat, C. R. L. Murthy, and S. Gopalakrishnan, "Acoustic emission source location in composite structure by Voronoi construction using geodesic curve evolution," Journal of the Acoustical Society of America, vol. 126, no. 5, pp. 2324-2330, 2009.

[22] W. Wevers and K. Lambrighs, "Applications of acoustic emission for structral health monitoring: a review," in Encyclopedia of Structural Health Monitoring, John Wiley \& Sons, Chichester, UK, 2009.

[23] J. Yick, B. Mukherjee, and D. Ghosal, "Wireless sensor network survey," Computer Networks, vol. 52, no. 12, pp. 2292-2330, 2008.

[24] P. Levis and D. Gay, TinyOS Programming, Cambridge University Press, Cambridge, UK, 2009.

[25] Y. Leng, D. Wenfeng, S. Peng, X. Ge, G. J. Nga, and S. Liu, "Study on electromagnetic wave propagation characteristics in rotating environments and its application in tire pressure monitoring," IEEE Transactions on Instrumentation and Measurement, vol. 61, no. 6, pp. 1765-1777, 2012.

[26] M. J. Blanch and A. G. Dutton, "Acoustic emission monitoring of field tests of an operating wind turbine," Key Engineering Materials, vol. 245-246, pp. 475-480, 2003.

[27] Á. Lédeczi, T. Hay, P. Völgyesi, D. R. Hay, A. Nádas, and S. Jayaraman, "Wireless acoustic emission sensor network for structural monitoring," IEEE Sensors Journal, vol. 9, no. 11, pp. 1370-1377, 2009.

[28] Y. Bao, J. L. Beck, and H. Li, "Compressive sampling for accelerometer signals in structural health monitoring," Structural Health Monitoring, vol. 10, no. 3, pp. 235-246, 2011. 

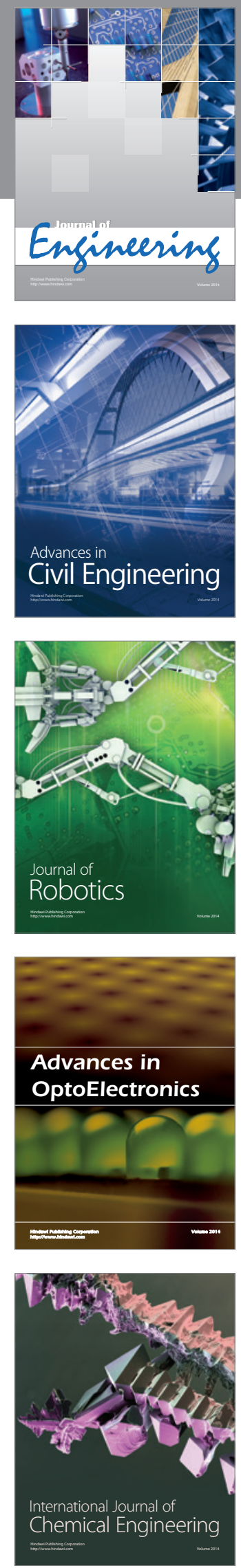

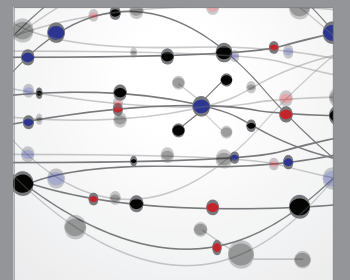

The Scientific World Journal
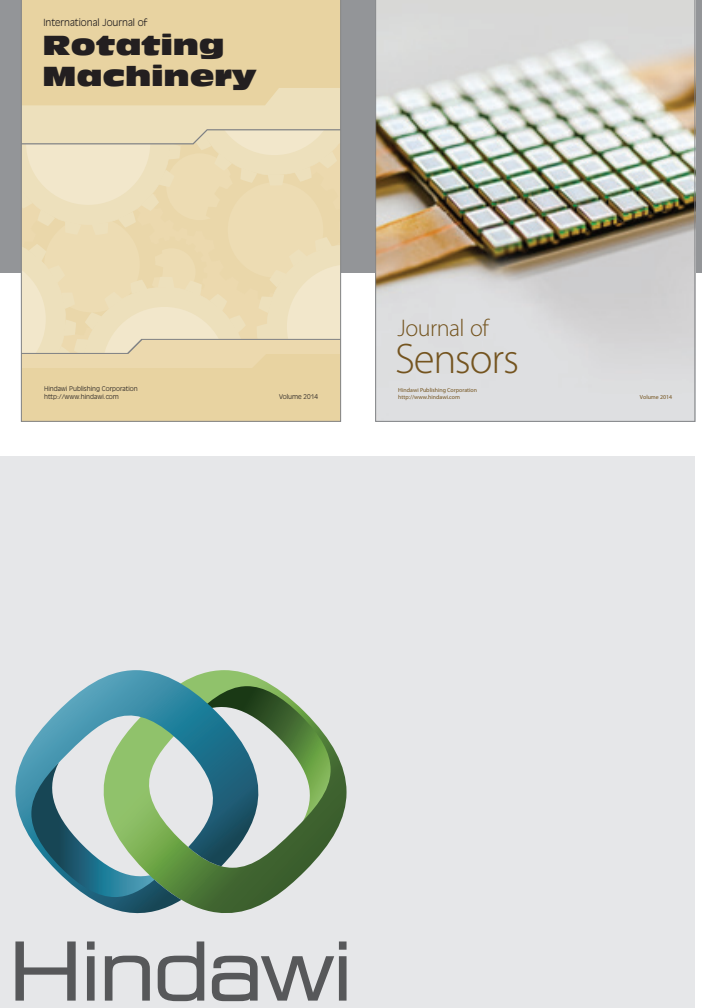

Submit your manuscripts at http://www.hindawi.com
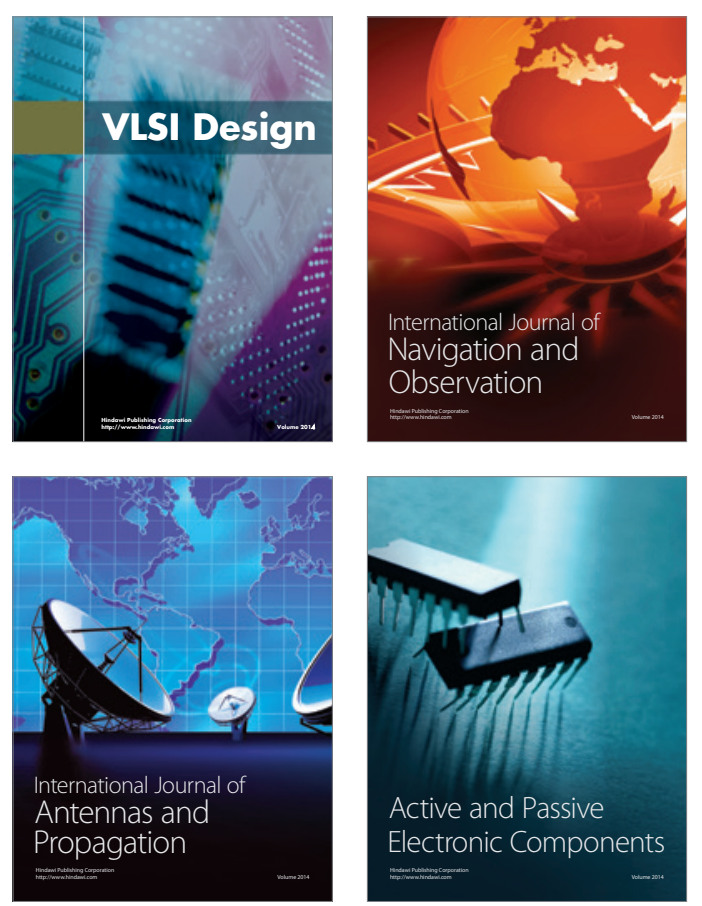
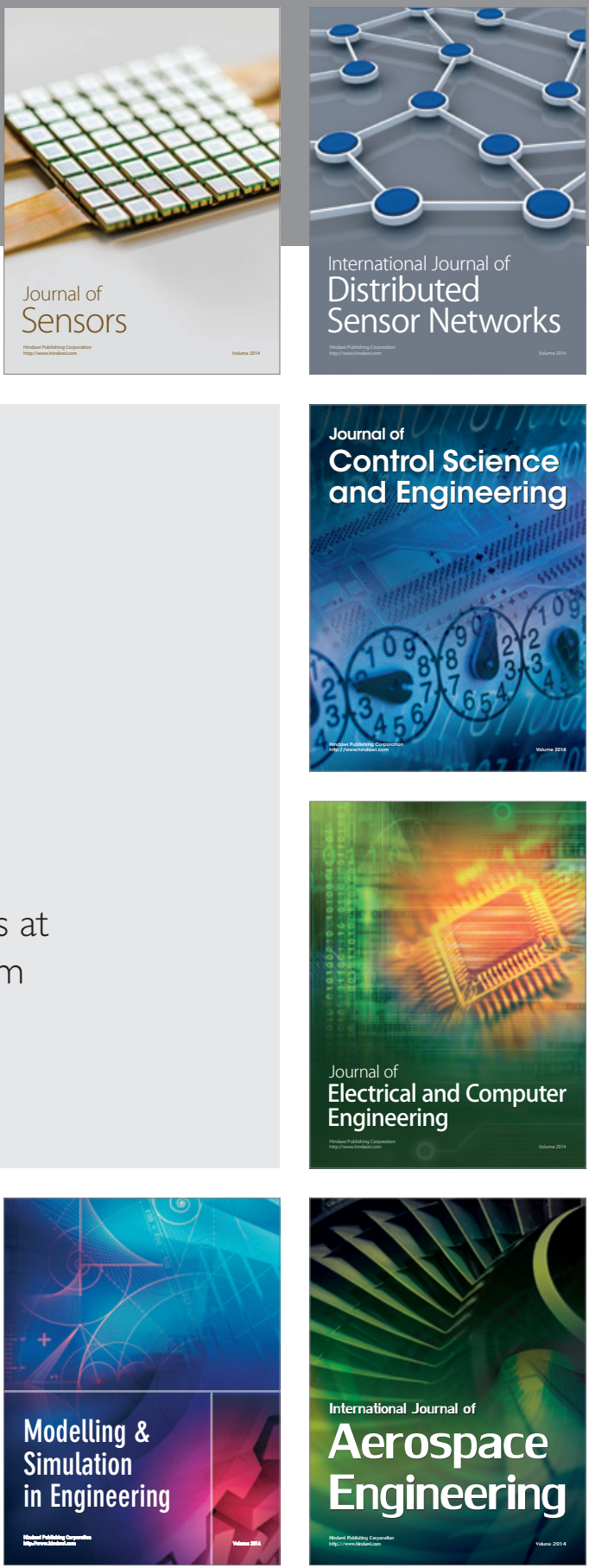

Journal of

Control Science

and Engineering
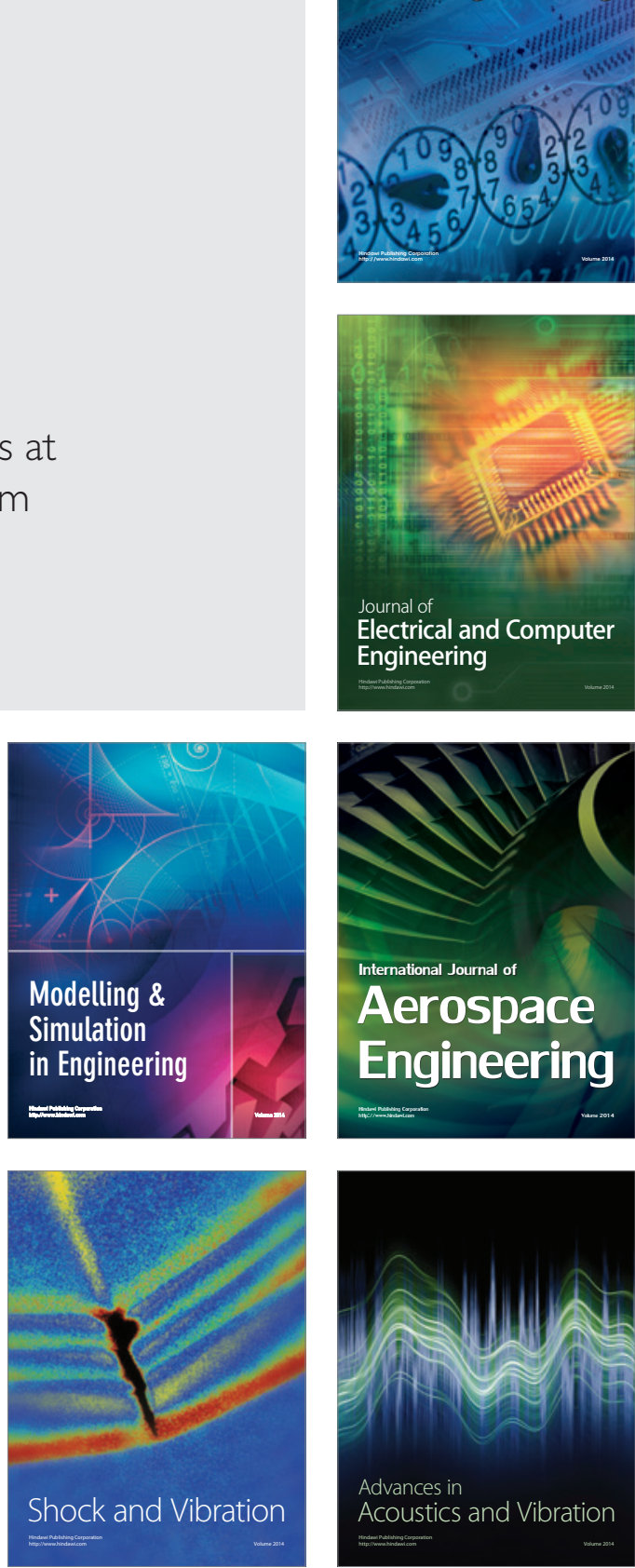